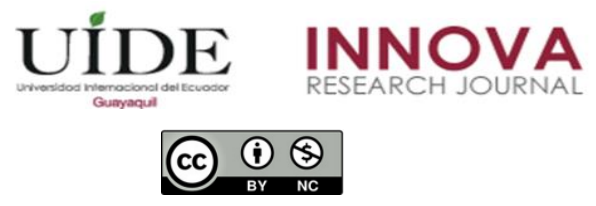

INNOVA Research Journal, ISSN 2477-9024

(Septiembre-Diciembre 2020). Vol. 5, No.3.2 pp. 249-271

DOI: https://doi.org/10.33890/innova.v5.n3.2.2020.1629

URL: http://revistas.uide.edu.ec/index.php/innova/index

Correo: innova@uide.edu.ec

\title{
El periodismo de investigación, un desafío en las aulas universitarias
}

\section{Investigative journalism, a challenge in university classrooms}

Dennys Jordán Correa

Gabriela Samaniego Rivas

Daybeth Gutiérrez Páez

Aldo Rivera Arreaga

Universidad Internacional del Ecuador, Ecuador

Autor para correspondencia: dejordanco@uide.edu.ec; gasamaniegori@uide.edu.ec;

dagutierrezpa@uide.edu.ec; alriveraar@uide.edu.ec

Fecha de recepción: 06 de noviembre de 2020 - Fecha de aceptación: 18 de diciembre de 2020

\section{Resumen}

La investigación constituye una de las tareas más complejas de la carrera periodística. Los futuros profesionales tienen serios desafíos para ajustarse a las exigencias del público y del sistema comunicacional. El presente artículo propone analizar y contrastar puntos de vista de varios autores, mostrar los resultados y formular propuestas a fin de lograr cambios que los encaminen a perfeccionar sus técnicas de investigación para obtener información más exacta sobre algún tema que propongan ajustándose a los requerimientos del medio. Las encuestas y entrevistas que acompañan este estudio realizado a profesionales, estudiantes y docentes de seis universidades de Guayaquil, tienen el fin de analizar si es correcta la forma en la que se imparte la materia de periodismo de investigación en las aulas universitarias y, si cabe la posibilidad de proponer sugerencias para mejorar la cátedra. Los medios tradicionales ya no realizan investigaciones periodísticas. No tienen las condiciones adecuadas para financiarlos, por eso buscan diferentes actividades que reemplacen la investigación, como noticieros, crónicas o coberturas de hechos que sean en el momento y no necesitan una investigación de fondo, según el medio de comunicación.

Palabras claves: Periodismo de investigación; universidades; estudiantes; docentes.

\begin{abstract}
Research is one of the most complex tasks of the journalistic career. Future professionals of journalism have serious challenges to adjust the demands of the public and the communication system. This article proposes to analyze and contrast the points of view of several authors, show results and formulate proposals in order to achieve changes that will lead them to perfect their research techniques to obtain more exact information on a topic that they propose, adjusting to the requirements of the environment. Surveys and interviews that accompany this study were carried out to professionals, students and teachers from six universities in Guayaquil, and have the purpose of analyzing if the way in which the subject of investigative journalism is taught correctly at
\end{abstract}


universities and, if possible, propose suggestions to improve the course of study. The traditional media no longer conduct journalistic investigations. They do not have adequate conditions for financing them, so look for different research activities to replace, such as news, stories or facts which are hedges at the time and do not require a background investigation, according to the media. Keywords: Investigative journalism; universities; students; evaluation; survey; teachers.

\section{Introducción}

\section{Conceptualización del Periodismo de Investigación}

El manual de la Organización de las Naciones Unidas para la Educación, la Ciencia y la Cultura, Unesco, señala que el Periodismo de Investigación es denominado como Emprendedor porque recolecta, analiza, organiza e interpreta las pruebas a partir de la propia iniciativa del informador, quien traspone varios obstáculos y riesgos con el objetivo de evidenciar asuntos subrepticios. Esto, generalmente ocurre por aquellos que, en posición de poder, restringen datos que son de interés público que afectan a la sociedad (Hunter, 2013). También se lo conoce como Periodismo de Rendición de Cuentas porque explica problemas complejos y pide razones a los poderosos; "es constructor de confianza y crea valor en la sociedad" (Starkman, 2014). Estas definiciones concuerdan con lo que Robert W. Green, fundador del Investigative Reporters / Editors (IRE), proponía como los 3 elementos consustanciales del Periodismo de Investigación: a) Trabajo propio del periodista, b) La relevancia social del tema, c) Que se trate de algo que se pretenda esconder (Reyes, 2005). Además, Green acotó: "Si el investigador no es periodista, no se puede hablar con propiedad como periodismo de investigación"; de allí que la responsabilidad y los principios éticos se exacerban en esta profesión.

El Periodismo de Investigación nace en Estados Unidos a finales del siglo XIX y a principios del siglo XX a partir de un grupo de periodistas conocidos como "los muckrakers" (rastrilladores de estiércol). Su denominación fue acuñada por el presidente Roosevelt a través de uno de sus discursos en el que se refería a un tipo especial de periodistas que "sólo sabían hurgar en lo negativo" para denunciar y escandalizar a la comunidad, principalmente sobre funcionarios públicos (Requejo, 2011). Ellos tenían un amplio espíritu de denuncia y ponían al descubierto los excesos del poder político, económico y religioso de la época para lograr un cambio social.

En Latinoamérica a fines de la década de los 70s, Colombia empezó a formar unidades de investigación denunciando actos de corrupción en los gobiernos. En los 80s, la tendencia se extendió por el resto de países. Por ejemplo, en Argentina los periodistas denunciaron las atrocidades de la dictadura militar y posteriormente la corrupción en el gobierno del presidente Menem. En Perú, la corrupción gubernamental y militar en los años de gobierno de Fujimori generó suficiente material para denunciar. En Ecuador, Juan Carlos Calderón, en su libro "Periodismo de Investigación", menciona que se realizaron importantes denuncias durante el gobierno de León Febres Cordero, de 1984. En los 90s se consolida al poder de la prensa y el Periodismo de Investigación goza su mejor época (Álvarez, 2015). 
Según un estudio publicado en "Los avatares del periodismo de investigación en Colombia" en el que se entrevistó a 32 periodistas investigativos colombianos, existen 3 tipos de generaciones desde los 70s (época del emblemático caso del Watergate) hasta la actualidad: 1) "Los pioneros" (1970 - 1980): denominados así por emprender iniciativas como: el Derecho de Petición de Información; realizar investigaciones de gran envergadura que resultaron en el enjuiciamiento de funcionarios públicos y la huida de banqueros. 2) "Los luchadores" (1990 2000): en aquella época los periodistas se enfrentan al auge de diversos obstáculos y peligros (secuestro, violación de sus derechos, bombas, censura, amenazas, exilio e incluso asesinato) para revelar sus denuncias: 3) "La nueva era" (2006 - actualidad): Son los periodistas de investigación más jóvenes que publican sus producciones utilizando material audio y visual por medio de las plataformas digitales, captando audiencias juveniles y manteniendo vigente la investigación. Incluso, en dicho estudio se mencionan los temas más relevantes para la comunidad: la corrupción y la política (44\%), el conflicto armado (24\%), medio ambiente (12\%), femicidio (6\%) y el resto trata otras problemáticas sociales (Lina Leal S. T., 2017).

\section{La ética, elemento fundamental en periodismo}

El ADN del periodismo es la ética, afirmada a través de una práctica profesional responsable e involucrada con la verdad, según la Organización de las Naciones Unidas para la Educación, la Ciencia y la Cultura (UNESCO, 2017). La ética abre paso a una ruta crítica del deber ser profesional, pero esta solo se entiende cuando está arraigado el significado del "compromiso con la sociedad" que constantemente va en busca de la excelencia dejando a un lado el apuro por publicar lo oculto o el afán de la primicia. "La información de calidad es la materia prima para la deliberación pública y, por lo tanto, para el ejercicio de la democracia" (Carrascal, 2013). El problema es que cada periodista y medio de comunicación suelen interpretar la ética conforme a su propia realidad, omitiendo las normas que, aunque no siempre están reglamentadas por escrito, se encuentran legisladas moralmente en el inconsciente de la sociedad y es allí cuando empieza el desafío de poner en práctica la ética sin necesidad de seguir un rígido manual.

Seguir estándares éticos no solo es lo correcto, sino que también protege la carrera y reputación del periodista; además aumenta el valor e impacto de las investigaciones. Aunque existen dificultades propias de la labor periodística, estas no pueden resolverse en la misma medida para todos los periodistas. Pulitzer enfatiza que por encima del conocimiento, de las noticias, de la inteligencia; el corazón y el alma del periódico descansan sobre su sentido moral (Lozano, 2013). Ese sentido que se compromete con la verdad, (Solís, 2007) deja claro que la responsabilidad social del periodista implica un absoluto respeto hacia los receptores quienes buscan información y opinión de profesionistas que realicen un trabajo apegado a los lineamientos éticos, los mismos que se forman desde las universidades y es allí cuando se involucra a los profesores.

El aprendizaje ético no solo se practica en el campo laboral, sino desde las aulas de clases. En una investigación realizada en los centros de educación superior de 14 países miembros de la Unión Europea, casi el 80\% de los maestros optan de una manera más clara por dar cabida a la ética dentro del periodismo, independientemente de solo perseguir un caso consideran incluso que las teorías de los grandes pensadores son necesarias para el futuro 
periodista. Como resultado consideran adecuado contar durante los primeros años de la malla curricular con un curso solo de ética filosófica, para fundamentar teóricamente la asignatura más específica de la deontología periodística. Se trata que los estudiantes posean más conocimientos y estén en mejores condiciones y adquieran nuevas competencias o habilidades para la toma de decisiones, emitir juicios y resolver dilemas de una manera razonada (Lozano, 2013).

La universidad por lo tanto se consolida como una base rígida, convirtiéndose en el entrenamiento adecuado para enfrentar a las intimidantes presiones sociales. Cabe recalcar que los principios "éticos" se interpretarán de manera diversa y variaran acorde a los intereses o negociaciones que influyen en el manejo de información de cada medio. Independientemente de alguna de estas situaciones con las que se encuentre el periodista, tiene prohibido la falsificación de documentos, suprimir o corregir informaciones y datos. El compromiso es informar hechos veraces sin manipulaciones que atenten contra la ética ya que "el periodismo de investigación ha sido desde siempre un arma política con la que destruir reputaciones y favorecer a partidos o políticos determinados" (Palancar, 2017). Es necesario aplicar el sentido común. Bivins citó en La ética y el derecho de la información que la obediencia a ciegas a cualquier filosofía nunca es suficiente si se realiza un análisis serio de una cuestión moral (Lozano, 2013).

Los medios tienen una alta dependencia económica de las empresas que publicitan sus productos y a consecuencia se ven limitados en la difusión de la información que pudiera estar relacionada con ellas, por tal razón si la fuente es de confianza, institucional, tiene mucho peso o existe apuro, no se contrasta (Villegas, Domínguez, y Martínez, 2009). Frente a esta realidad, la precariedad laboral, la calidad de la información se ven afectadas y finalmente se copia y se pega de acuerdo a los intereses de quienes están al poder, Restrepo citó que en "Latinoamérica hay dos clases de periódicos. Los que pertenecen a grupos familiares y los que son propiedad de grupos empresariales y políticos. Los que más confianza despiertan son los primeros, porque asumen el periodismo como un reto" (Carrascal, 2013)

En una entrevista en España realizada a periodistas andaluces con una edad media de 36 años, de cada cinco, cuatro redactores y un directivo dentro del medio, en su mayoría coincidieron que se está perdiendo "a pasos agigantados" el poder crítico del periodismo para convertirse en un escaparate de los intereses económicos y políticos dominantes en la sociedad (Villegas, Domínguez, y Martínez, 2009). El contraste, el rigor, la honestidad y la calidad son cuatro elementos fundamentales que se están perdiendo y atentan contra la credibilidad; sin embargo, no sólo la calidad de la información se ve perjudicada por estas situaciones laborales, sino que también se ve comprometida la ética de los periodistas causando el deterioro de su imagen; cada periodista debe valorar su realidad y la de su medio para decidir qué es lo que está bien y qué es lo que está mal, en su ambición por hacer un periodismo ético.

\section{Casos representativos del periodismo de investigación}

A inicio de la década de los setenta surgieron escándalos de corrupción que mancillaron la carrera política de varios funcionarios públicos e incluso de los altos mandos del gobierno norteamericano. El periodismo de investigación fue el gran protagonista al filtrar datos de interés público como los Papeles del Pentágono y Watergate, los más representativos en la historia de 
esta profesión. La publicación de estos reportajes trascendió fronteras, comprobando la importancia que tiene este tipo de ejercicio para la sociedad como es la vigilancia del poder político y económico; de esta manera se corrobora que la característica relevante de esta labor es "la actitud crítica ante los políticos" (Muraro, 1997).

Desde entonces la investigación periodística ha transcendido en el ámbito internacional gracias a las nuevas tecnologías de información y comunicación (TIC) que permite a los responsables de este oficio conectarse con comunicadores de otras fronteras produciendo así una ciencia global que desmantele la corrupción en un nivel transnacional. "La creación de redes de periodistas y el trabajo en equipo es fundamental, ya que puedes parar una historia en un país, pero no puedes pararla en todo el mundo" (Walker, 2015). De allí radica la importancia de informar a la sociedad de todo aquello que se quiera mantener en sigilo con una opinión crítica, reflexiva y amparada siempre en la libertad de expresión.

Si bien el término 'crítico' puede ser considerado como la formulación de un juicio tanto positivo como negativo, en la práctica este género consiste en comunicar al lector aspectos de la vida pública que los dirigentes partidarios o los funcionarios preferirían mantener bajo reserva (Muraro, 2000).

El develamiento de casos de corrupción como: Los Papeles del Pentágono (1971), Watergate (1972), WikiLeaks (2006), Walmart México (2012) Los Papeles de Panamá (2016) y Odebrecht (2016); aportaron reflexiones significativas para la opinión pública. En su Manual de Periodismo, Vicente Leñero y Carlos Marín explican que el reportaje es "el género mayor del periodismo, el más completo" (Leñero y Marín, 1986).

\section{Papeles del Pentágono y Watergate}

¿El periodismo de investigación puede desequilibrar la vida política de la más alta autoridad de un país? ¡Sí!, o al menos eso se demostró en la década de los 70. En 1971, periodistas del New York Times divulgaron información de gran confidencialidad protegida por el Departamento de Defensa de EE. UU sobre la cuestionada participación de ese país en la Guerra de Vietnam (1945 y 1967). "Los Papeles del Pentágono", se denominó a la revelación de 7000 copias que mostraron cómo se mintió sistemáticamente, no sólo a la opinión pública sino también al congreso desde la administración de Johnson (1963 - 1969) sobre la cuestionada intervención militar. Esto produjo controversia pública, social y judicial, desestabilizando al gobierno de Nixon, pero el detonante sucedió meses más tarde. En 1972, cinco hombres portando equipo fotográfico e instrumentos electrónicos fueron detenidos por la policía cuando circulaban dentro de las oficinas Watergate, en Washington, sede del Comité Nacional Demócrata. Este inusual hecho despertó la curiosidad de los periodistas Carl Bernstein y Bob Woodward, del diario The Washington Post; esto originó a una exhaustiva investigación en donde, aparentemente, altas autoridades del gobierno estaban involucradas, incluyendo al propio presidente. El 8 de agosto de 1974, por primera vez, en la historia política del país norteamericano un presidente dimitía tras probarse que Richard Nixon, líder de los 
Republicanos, filtró espías en Watergate, tal vez con el objetivo de obtener algo que lo beneficie para su reelección.

Es triste y trágico cuando el líder de un país tiene que abandonar su puesto como en este caso... Es deprimente y la gente va por las calles como desilusionada. Esto ha sido mostrar el lado bueno y malo de Norteamérica. Pero el lado bueno ha sido, también, una demostración de que el sistema funciona (Woodward, 2016).

\section{Filtración de datos: Wikileaks}

En el año 2006, la transformación de la comunicación ya estaba ligada al desarrollo del internet y eso facilitó la revelación de información oculta que se pudo volver pública gracias a los ataques cibernéticos. Wikileaks, página web creada por el australiano Julian Assange, fue el mayor sitio de filtración de datos de carácter confidencial desde que empezó su actividad en el 2006 hasta el 2010, cuando publicó 92000 documentos sobre la guerra de Afganistán, 2500 cables secretos de la diplomacia de EE.UU., entre otros datos de estricta reserva. Pero, ¿qué consecuencias tuvo estas revelaciones? para su fundador, una orden de detención por parte de la Interpol "Julian Assange con Wikileaks puso en evidencia que en nuestra sociedad informatizada el poder se sostiene en la información que obtiene, produce y administra, siendo un territorio de lucha política" (Guzmán, 2016); y para la sociedad, reconocer que el derecho a la información es parte de las libertades ciudadanas, pero el Estado puede restringirlo a favor de la defensa de derechos. Wikileaks pudo ampararse a las garantías de prensa, pero también tenía que cumplir códigos éticos que eviten publicar hechos no corroborados, procedimientos que se encuentran en el periodismo tradicional y de investigación, siendo una de las razones por las que este sitio web recurrió a medios de comunicación ya que en algún punto fue necesario transformar toda la información obtenida en historias y noticias que requerían criterios periodísticos. Posteriormente "Assange fue asilado por Ecuador en su Embajada en Londres en 2012, cuando ingresó bajo el argumento de que temía ser arrestado en suelo británico y deportado a EE.UU., donde podría ser juzgado por la publicación de documentos militares y diplomáticos clasificados" (González, 2019).

\section{Walmart México}

Otro de los casos de actividades fraudulentas que ha merecido diversas publicaciones en medios nacionales e internacionales es el de Walmart- México gracias a un ex trabajador de la subsidiaria en el país azteca, que denunció una estrategia de sobornos por más de 24 millones de dólares para construir múltiples tiendas en todo el país. Con un reportaje bastante completo, Daniel Barstow escribe para el New York Times en abril de 2012 sobre la llamada "cultura de corrupción" y muestra un ejemplo de trabajo informativo de calidad. La importante investigación del diario norteamericano encontró evidencias con más de 16 fuentes directas, documentales, entrevistas y reportes sobre coimas y la expansión de la empresa en México, donde se ofrecía empleo a 209000 ciudadanos, dato que lo convierte en "el empleador privado más grande del país" (Fanger, 2012). ¿Pero cuáles son las repercusiones éticas de este caso? Según un informe de Transparencia Internacional, el índice de percepción de la corrupción del órgano para el año 2011, señala que los gobiernos en América Latina, Asia y Medio Oriente son “incapaces de 
proteger a sus ciudadanos del abuso cometido contra recursos públicos, sobornos y decisiones tomadas en secreto" (Wharton, 2012). En cambio, en el ámbito periodístico resulta una historia con mucha información que sintetizar y analizar, principalmente, sobre estrategias empresariales y sus actos corruptos.

\section{Periodismo Global: Los Papeles de Panamá y Odebrecht}

Desde el 2016 en América Latina se evidenciaron dos casos significativos de corrupción: Los Papeles de Panamá y Odebrecht. Pero, ¿cuál fue su impacto y conclusiones que dejaron estas publicaciones en la sociedad?

Este año un equipo de 190 periodistas de 65 países, integrantes del Consorcio Internacional de periodistas de Investigación (ICIJ), filtraron datos que revelaron las cuentas bancarias en paraísos fiscales de personajes reconocidos (reina Isabel II, Leonel Messi, Mauricio Macri...) e incluso mancilló a una de las constructoras más importantes de América Latina "Odebrecht S.A." ligada a sobornos a políticos y funcionarios del gobierno para la obtención de más de 100 contratos en 12 países como (Venezuela, Panamá, Perú, México, Ecuador, Estados Unidos, Colombia, Argentina...). Entre los años 2001-2016 la empresa recibió USD 3.336 mil millones por coimas, según una investigación del departamento de Justicia de Estados Unidos.

La corrupción es uno de los medios para atentar en contra de la ética que directamente afecta al gobierno corporativo de los países y de las empresas, poniendo en entredicho la actuación y las decisiones tomadas por las partes involucradas Fuente especificada no válida.

¿Por qué estos personajes públicos y privados estaban involucrados en esta red de corrupción? Las principales causas son obtener poder y control, ambiciones que los conducen a lucrarse de las arcas fiscales desestabilizando la situación socio-económica de una nación.

\section{El rol de la Academia en la investigación periodística}

Realizar periodismo de investigación es una tarea ardua, requiere de una serie de pasos que llevan a obtener un producto en que la hipótesis inicial puede cambiar y la investigación necesite más tiempo y recursos, por eso el análisis de estos casos se ampara en tres aspectos:

1. La mayoría de investigaciones han sido respaldadas por medios de alta credibilidad y por profesionales en la comunicación cuya premisa ha sido la búsqueda de la verdad en una historia.

2. Todos han requerido financiamiento.

3. El olfato periodístico se vuelve fundamental, toma tiempo desarrollarlo; por eso, la mejor forma de generar este tipo de iniciativas debe comenzar desde la práctica universitaria.

Esta especialidad se caracteriza porque "la realiza el periodista y no la justicia, la policía o particulares interesados. Es decir, es una investigación que parte por la iniciativa propia del 
periodista y que es desarrollada plenamente por él o ella" (Santoro, 2004). En una entrevista a profesionales de la comunicación en planteles de educación superior en Guayaquil, se evidencia que el periodismo de Investigación debe inculcarse desde las aulas con herramientas teóricoprácticas que fortalezcan la experticia de todo comunicador.

Para el autor The Reporter's Handbook "la superficialidad que caracteriza a muchos diarios se debe a que los periodistas no tienen la capacidad de localizar, entender y utilizar gran número de documentos y datos estadísticos a fin de abonar a una hipótesis" (Campbell, 1994), de allí radica la importancia de empoderar a los futuros profesionales dentro de las aulas. Este criterio lo comparten docentes que imparten la cátedra de Investigación Periodística en las universidades Casa Grande, Católica Santiago de Guayaquil, Universidad de Especialidades Espíritu Santo y Universidad de Guayaquil.

\section{Tabla 1}

Análisis del periodismo de investigación como una especialización que debe inculcarse desde las aulas

\begin{tabular}{|c|c|c|c|}
\hline $\begin{array}{l}\text { Lcda.Mariuxi León } \\
\text { (UCSG) }\end{array}$ & $\begin{array}{l}\text { Lcda. Sylvia } \\
\text { Poveda } \\
\text { (UEES) }\end{array}$ & $\begin{array}{c}\text { Lcdo. Rubén } \\
\text { Montoya } \\
\text { (Casa Grande) }\end{array}$ & $\begin{array}{l}\text { Lcda. Yamil de Haz } \\
\text { Cruz } \\
\text { (Facso) }\end{array}$ \\
\hline $\begin{array}{l}\text { Más allá del carácter } \\
\text { de que sea } \\
\text { especialización o no, } \\
\text { también se debe pensar } \\
\text { siempre que el } \\
\text { periodismo de } \\
\text { investigación molesta } \\
\text { al poder, por lo tanto, } \\
\text { como rama es posible } \\
\text { que eso también sea } \\
\text { frenado desde el } \\
\text { Estado, no solo porque } \\
\text { han eliminado las } \\
\text { especialidades en } \\
\text { general dentro de las } \\
\text { carreras, sino también } \\
\text { porque el periodismo } \\
\text { de investigación es } \\
\text { visto como una } \\
\text { instancia de peligro. }\end{array}$ & $\begin{array}{l}\text { Debe ser una } \\
\text { especialización } \\
\text { dentro de la carrera } \\
\text { porque el periodista } \\
\text { debe saber capturar } \\
\text { los hechos del día a } \\
\text { día, pero también } \\
\text { necesita trabajar en } \\
\text { lo que es } \\
\text { profundidad, debe } \\
\text { dominar los dos } \\
\text { campos, en algunos } \\
\text { hechos se necesita } \\
\text { esa inmediatez, } \\
\text { pero en otros } \\
\text { ofrecer una } \\
\text { reflexión al público } \\
\text { y ese sería el } \\
\text { periodismo de } \\
\text { investigación. }\end{array}$ & $\begin{array}{l}\text { Es una materia } \\
\text { que tiene que estar } \\
\text { en la malla } \\
\text { superior, no en } \\
\text { primero o segundo } \\
\text { ciclo, requiere } \\
\text { algunas } \\
\text { herramientas que } \\
\text { tengan los } \\
\text { estudiantes, unos } \\
\text { conocimientos } \\
\text { previos. }\end{array}$ & $\begin{array}{l}\text { El Periodismo de } \\
\text { Investigación abarca una } \\
\text { mayor arista, es el que } \\
\text { lleva un mejor } \\
\text { componente porque ya } \\
\text { los estudiantes han } \\
\text { tenido un preámbulo, un } \\
\text { conocimiento. }\end{array}$ \\
\hline
\end{tabular}

Nota: Entrevista a docentes de diferentes universidades de la ciudad de Guayaquil. 
La cátedra de Periodismo de Investigación es una disciplina que sí debe formar parte de la malla académica de los centros de educación superior que forman a los futuros comunicadores, esto opinan todos los maestros que imparten la asignatura en las cuatro universidades citadas en el párrafo anterior.

\section{Figura 1}

Terminología basada en la especialización

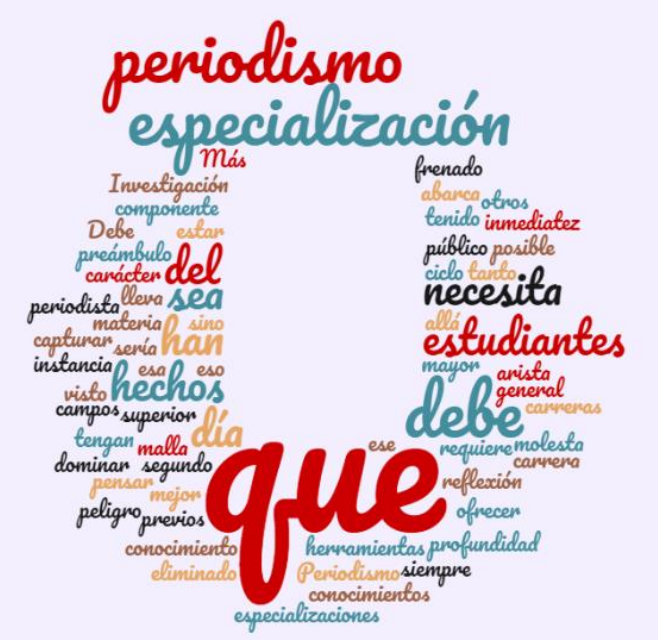

En la subsecuente tabla de opiniones se puntualizan algunas competencias intrínsecas y extrínsecas que debe tener todo periodista, aquellas que se desarrollan durante un determinado tiempo y que a los estudiantes les convendría poseer. La Real Academia Española define al término como pericia, aptitud o idoneidad para hacer algo o intervenir en un asunto determinado". Por tal motivo, al hablar de competencias es indiscutible analizar aquellas que requiere esta profesión, los catedráticos señalan que el uso adecuado de fuentes, la eficiencia, veracidad, curiosidad, excelente redacción y el manejo apropiado de herramientas se convierten en la parte medular para el ejercicio de la profesión.

\section{Tabla 2}

Principales competencias del periodista

\begin{tabular}{clll}
\hline $\begin{array}{c}\text { Lcda. Mariuxi León } \\
\text { (UCSG) }\end{array}$ & \multicolumn{1}{c}{$\begin{array}{c}\text { Lcda. Sylvia Poveda } \\
\text { (UEES) }\end{array}$} & $\begin{array}{c}\text { Lcdo. Rubén Montoya } \\
\text { (Casa Grande) }\end{array}$ & $\begin{array}{c}\text { Lcda. Yamil de Haz } \\
\text { Cruz } \\
\text { (Facso) }\end{array}$ \\
\hline $\begin{array}{c}\text { Delimitar y reconocer } \\
\text { temáticas y objetivos de } \\
\text { investigación. }\end{array}$ & $\begin{array}{l}\text { El periodista debe } \\
\text { conocer herramientas } \\
\text { digitales para potenciar }\end{array}$ & $\begin{array}{l}\text { Todo profesional en } \\
\text { comunicación debe saber } \\
\text { el manejo de } \\
\text { herramientas, tener }\end{array}$ & $\begin{array}{c}\text { El periodista debe ser } \\
\text { claro, veraz, oportuno, } \\
\text { eficiente. }\end{array}$ \\
$\begin{array}{c}\text { la difusión, estar } \\
\text { documentales, personales... }\end{array}$ & $\begin{array}{l}\text { informado y tener } \\
\text { curiosidad, tiempo y una }\end{array}$ & $\begin{array}{l}\text { curiona redacción. } \\
\text { estructura ética sólida. }\end{array}$ \\
editar. & & & \\
\hline
\end{tabular}

Nota: Entrevista a docentes 
Las opiniones de los catedráticos concuerdan que esta asignatura: Investigación Periodística, debe incorporarse en la malla académica de todas las instituciones de educación superior porque sin duda esto forjará mejor las competencias de los comunicadores.

\section{Figura 2}

Terminología basada en las competencias requeridas en periodismo

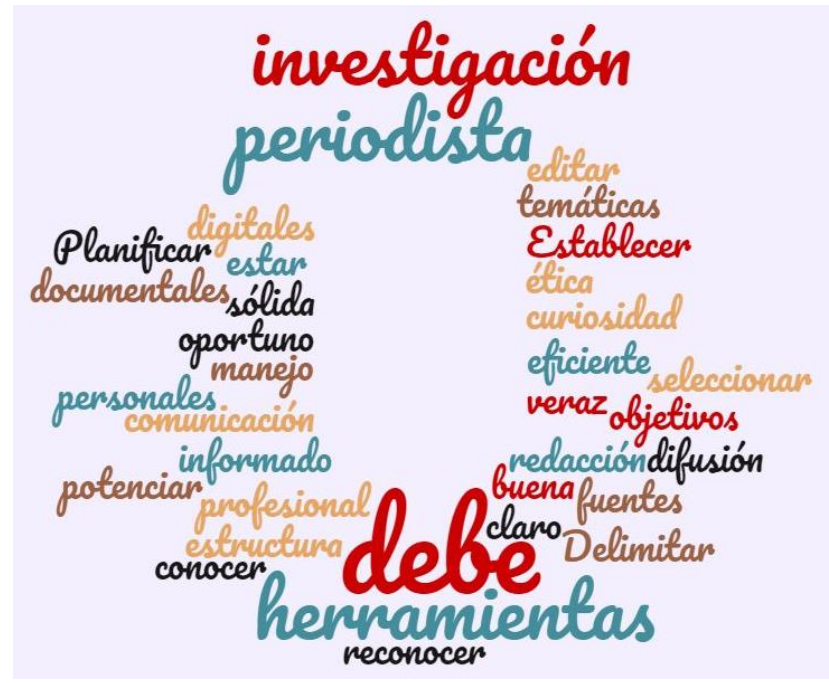

Las TIC permiten realizar alianzas con medios de comunicación que operan en otros territorios para abordar temas que afectan a distintos países. En abril de 2018, algunos medios establecieron cadenas para compartir datos sobre todo lo que ocurría en la frontera entre Ecuador y Colombia con respecto a los atentados causados por disidentes de las Farc y luego el secuestro y posterior asesinato de tres periodistas del Diario El Comercio.

En ese momento el periodismo de investigación se logró a través de la unión de periodistas investigativos de dos países de Ecuador y Colombia, no sólo nos quedamos con lo que decían las autoridades sino como tienes estas conexiones "cadenas amigas" en otras partes del mundo te permiten compartir información porque quieres analizar un tema que compete no sólo a tu país sino a la región, es algo que también se está convirtiendo en tendencia hoy (Valarezo, 2018).

Sin embargo, para garantizar el adecuado ejercicio de los futuros profesionales de esa área de la comunicación se necesita mejorar el contenido de la asignatura y a la vez incluir más proyectos de investigación periodística para reafirmar la especialización. 


\section{Tabla 3}

Proyectos de Investigación periodística que realizan por semestre

\begin{tabular}{|c|c|c|c|}
\hline $\begin{array}{l}\text { Lcda. Mariuxi León } \\
\text { (UCSG) }\end{array}$ & $\begin{array}{l}\text { Lcda. Sylvia } \\
\text { Poveda } \\
\text { (UEES) }\end{array}$ & $\begin{array}{c}\text { Lcdo. Rubén } \\
\text { Montoya } \\
\text { (Casa Grande) }\end{array}$ & $\begin{array}{l}\text { Lcda. Yamil de } \\
\text { Haz Cruz } \\
\text { (Facso) }\end{array}$ \\
\hline $\begin{array}{l}\text { Hay un trabajo que se } \\
\text { está desarrollando en } \\
\text { investigación sobre } \\
\text { Violencia contra la } \\
\text { mujer, se está } \\
\text { trabajando con los } \\
\text { estudiantes, y con } \\
\text { organizaciones de la } \\
\text { sociedad civil. }\end{array}$ & $\begin{array}{l}\text { En esta materia se } \\
\text { trabaja con un } \\
\text { proyecto de } \\
\text { investigación lo que } \\
\text { duraría un período } \\
\text { académico, lo que a } \\
\text { veces es muy poco. }\end{array}$ & $\begin{array}{l}\text { Dentro de la } \\
\text { materia no está } \\
\text { previsto que ellos } \\
\text { hagan un trabajo de } \\
\text { campo en ese sentido }\end{array}$ & $\begin{array}{l}\text { En la materia de } \\
\text { periodismo de } \\
\text { investigación por lo } \\
\text { menos unos cinco o } \\
\text { seis proyectos los } \\
\text { jóvenes realizan en el } \\
\text { semestre... }\end{array}$ \\
\hline
\end{tabular}

Nota: Entrevista a docentes

Incluir más proyectos de investigación es lo que coinciden todos los catedráticos, principalmente en los últimos niveles de la carrera. Según Poveda, en ese lapso ya los futuros profesionales tendrían fortalecidos los conocimientos y habilidades que demanda esta profesión. Sin embargo, no en todas las instituciones educativas encuestadas se encuentran trabajando en proyectos de investigación con los estudiantes debido al tiempo que demanda este tipo de investigaciones.

Yo creo que debe ser una especialización dentro de la carrera, porque el periodista debe saber capturar lo hechos del día a día, pero también necesita trabajar en lo que es profundidad, debe dominar los dos campos, en algunos hechos nosotros necesitamos esa inmediatez, pero en otros necesitamos ofrecer una reflexión al público y ese sería el periodismo de investigación (Poveda, 2018).

A continuación, se ha recopilado la información de encuestas realizadas a los docentes que ejercen esta asignatura con el fin de obtener datos más reales sobre la situación académica de los centros de educación superior más distintivos de la ciudad de Guayaquil.

El Periodismo de Investigación es una asignatura que sí debe formar parte de la malla académica de todas las universidades opinan el 100\% de los docentes encuestados. 


\section{Figura 3}

\section{Competencias de los Comunicadores}

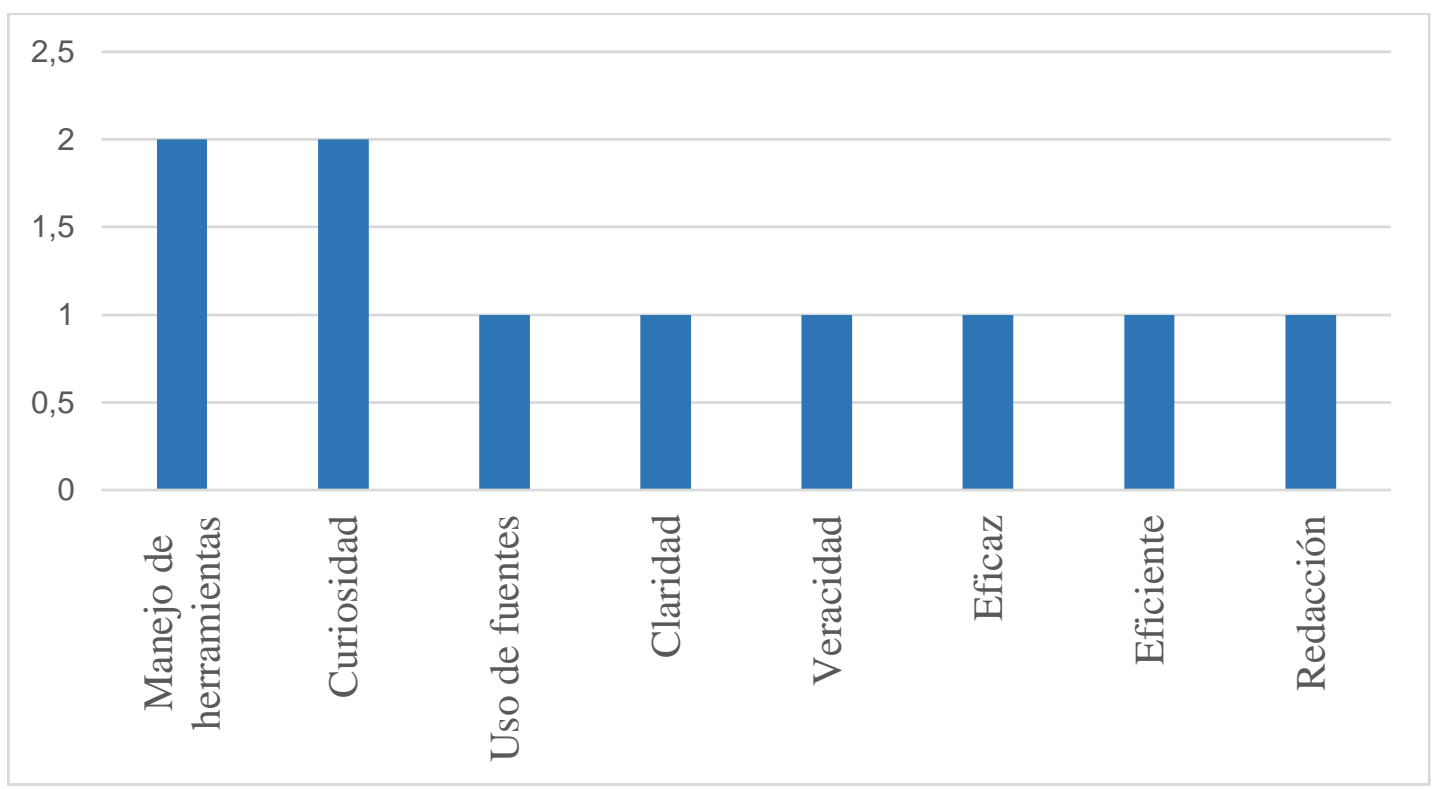

En el actual mundo de la comunicación los nuevos desafíos hacen que el periodista se acople a un escenario de adaptación continua. Más aún, si la imparable tendencia que muestran hoy los medios hacia la concentración de la homogenización de los mensajes va en crecimiento (Llanca y Reyes, 2017). Ante esta situación el periodismo de investigación se convierte en la primera opción para cambiar este panorama ya que su rigurosidad en el tratamiento de la información forja mensajes especializados, además que desarrolla habilidades como la exploración, persistencia, equilibrio, talento creativo y de búsqueda (Soto, 2013). De allí la importancia de consolidar las bases de un buen periodismo en las aulas universitarias porque la mayoría de los estudiantes tienen una percepción del profesional que se basa en descubrir la verdad y ponerla en conocimiento del público; sin embargo, en la práctica no pueden probar la amplitud de este término.

En la realidad este concepto es mucho más complejo y requiere de particularidades específicas. "En los estudios realizados para determinar características se utilizan mecanismos como la observación participante y la etnografía, que pretenden abordar in situ la práctica de la profesión" (Correa, 2013). Este último fenómeno se convierte en el principal déficit que tienen a menudo las universidades porque alejan de la práctica estos dos últimos componentes que son indispensables para el desarrollo de un verdadero proyecto de investigación; un claro ejemplo presentaron los estudiantes de la Facultad de Comunicación de la Universidad Internacional del Ecuador, quienes ejecutaron el "Proyecto de Inserción Social y Educativa para reforzar la comunicación comunitaria en Barrios de Paz", un lugar vulnerable de la ciudad de Guayaquil, ubicado en la Isla Trinitaria. 
"El proyecto tuvo como prioridad buscar participantes para impartir herramientas necesarias que permitan comunicar, de manera propia, todos aquellos eventos que consideren relevantes dentro de su comunidad; por eso, a través de la clase Investigación Participante se combinó la investigación social y sus distintos métodos, el trabajo de campo en donde se forja con el modelo sujeto-sujeto y la acción dentro de la comunidad" (Orellana, 2018).

\section{Desafíos de los docentes}

Uno de los principales desafíos para los docentes es que los estudiantes aprendan sobre el terreno, es decir desarrollar clases participativas que involucren realizar trabajos que sean publicables, para que lo aprendido no se quede en una simple calificación (Llanca y Reyes, 2017). Es por eso que el docente cumple un rol importante en el proceso de aprendizaje prácticoteórico como se indica en la investigación. El laboratorio de periodismo. La práctica universitaria de la idea fundacional de Pulitzer: "la razón es que el periodismo es una carrera con un alto componente práctico, se trata pues, de orientar el trabajo de los estudiantes hacia la producción periodística en todas sus modalidades. De enseñar y aprender periodismo ejerciéndolo, en circunstancias reales" (Correa, 2013).

Para el presente estudio se consideraron las opiniones de los estudiantes que cursan los últimos semestres de la carrera de Periodismo de seis universidades de la ciudad de Guayaquil; de esta manera se evaluaron las nociones adquiridas en el área de periodismo de investigación.

\section{Figura 4}

Número de materias relacionadas con periodismo de investigación que se presentan en su malla académica
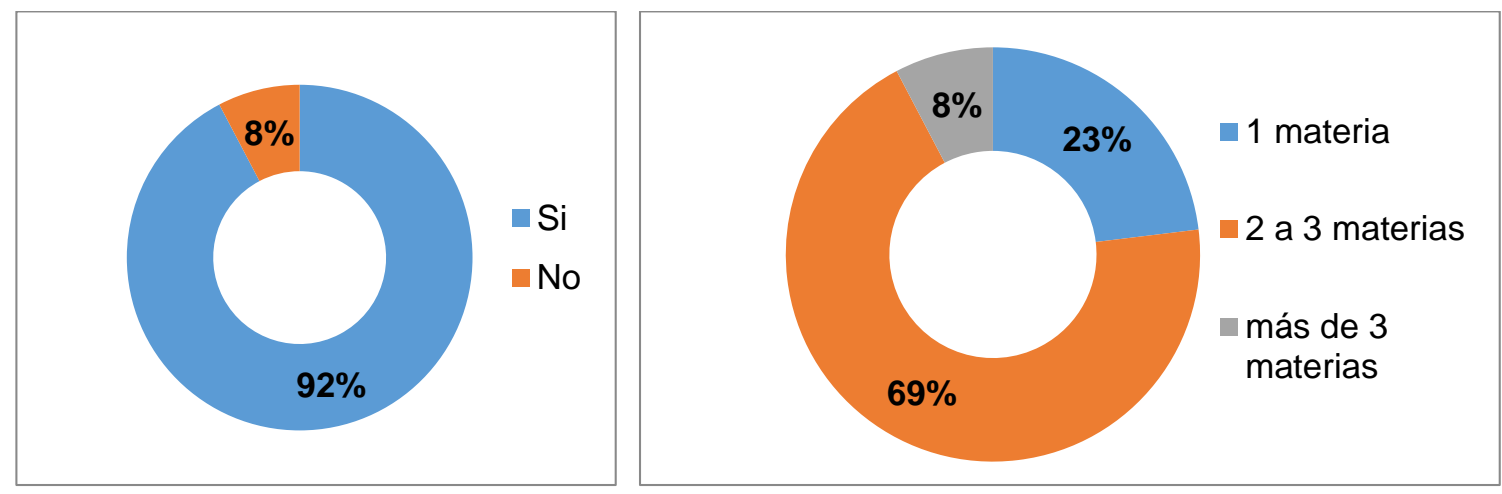

El 92\% de los encuestados opinan que en su malla curricular si cuentan con materias relacionadas al periodismo de investigación, mientras que el $8 \%$ afirman lo contrario. De este rubro, el $69 \%$ de los encuestados coinciden en que el número de materias relacionadas a 
investigación periodística se encuentra en el rango de 2 a 3 materias, mientras un $23 \%$ indica que recibieron 1 materia y solo un $8 \%$ mencionaron que vieron más de 3 materias.

\section{Figura 5}

Utilidad de los contenidos impartidos en las asignaturas

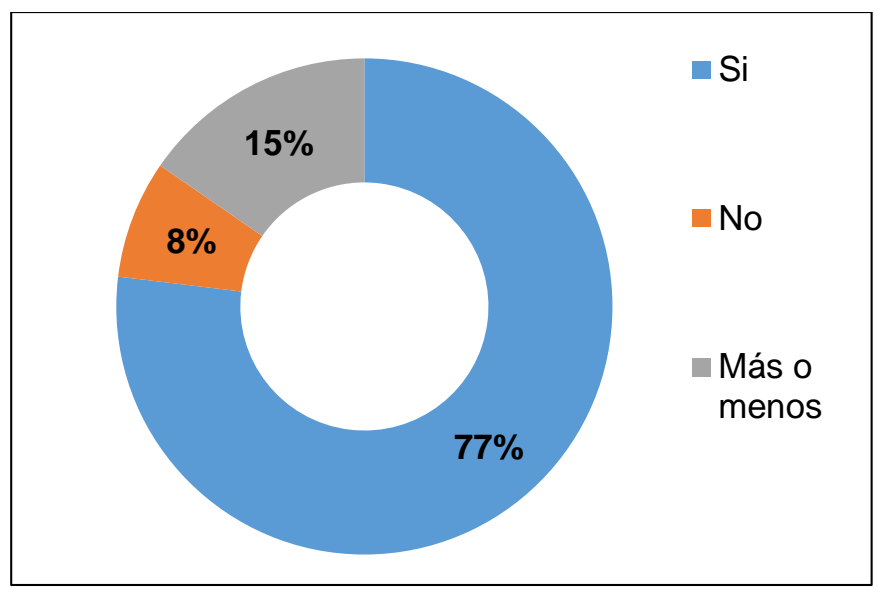

El 77\% de los encuestados coinciden en que el contenido impartido en la materia de investigación periodística si ha sido útil mientras que un $15 \%$ indica que más o menos y el $8 \%$ menciona que no ha sido útil.

Ante este panorama es indispensable que el profesor incorpore nuevas estrategias en su labor de enseñanza "El docente es, sin duda, un sujeto 'constructivista' que continuamente construye, elabora y prueba su teoría del mundo. Esto es consistente con la visión actual de la enseñanza considerada como una actividad del pensamiento profesional" (Díaz, 2009). Por lo que es necesaria la actualización constante de conocimientos, así como la práctica. Tal es el caso de la Universidad de Cartagena donde los docentes aún mantienen viejos modelos pedagógicos combinados con breves contenidos y poca experiencia. Así, el estudiante únicamente se limita a consultar información. (Vega y Restrepo, 2015).

De allí la necesidad de hacer clases prácticas, elaborar talleres, hacer pasantías, trasladarse a una sala de redacción, tener la asesoría de profesionales que laboren en medios de comunicación con conocimientos actualizados en el área de estudio. 


\section{Figura 6}

Tipo de proyectos de investigación realizados
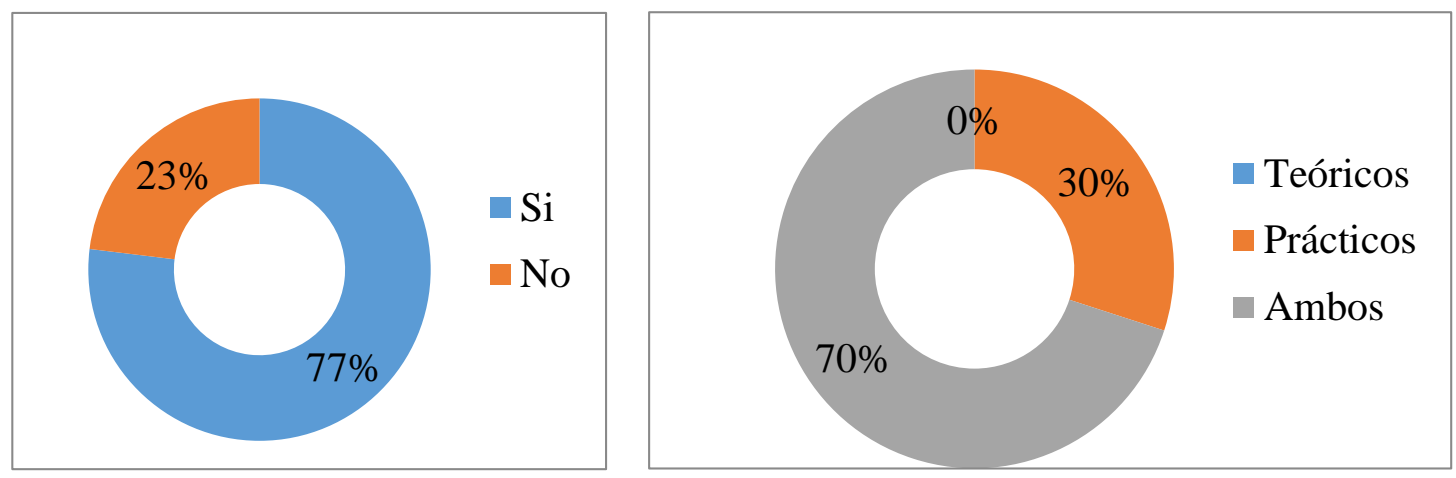

El 77\% de los encuestados coinciden en que si han realizado proyectos que involucren las nociones de investigación periodística aprendidas, mientras que un $23 \%$ indica que no ha realizado ningún trabajo relacionado a esta rama del periodismo. De este rubro el $70 \%$ de los encuestados coinciden en que los proyectos de investigación realizados fueron tanto prácticos y teóricos, mientras que un $30 \%$ indica que dichos proyectos fueron totalmente prácticos y un $0 \%$ teóricos.

\section{Planes de estudio y los requisitos del medio en Ecuador}

Globalmente, existe controversia entre la formación propuesta por los académicos y los medios de comunicación. Tomando en cuenta este antecedente, los medios españoles establecieron un marco basado en el reconocimiento de perfiles profesionales, en el que se toma en cuenta requisitos fundamentales de la academia y desarrollo de competencias. "El Libro Blanco de Títulos de Grado en Comunicación destaca dos objetivos: por un lado, competencias profesionales de recopilación, selección y transmisión de información en los diferentes géneros y formatos periodísticos. Por otro, lo que Reese ha llamado «habits of mind», conocimientos de pensamiento que capaciten para informar, analizar e interpretar los acontecimientos sociales y políticos" (Humanes y Roses, 2014). 


\section{Figura 7}

El conocimiento adquirido ha desarrollado competencias para laborar en un medio de comunicación

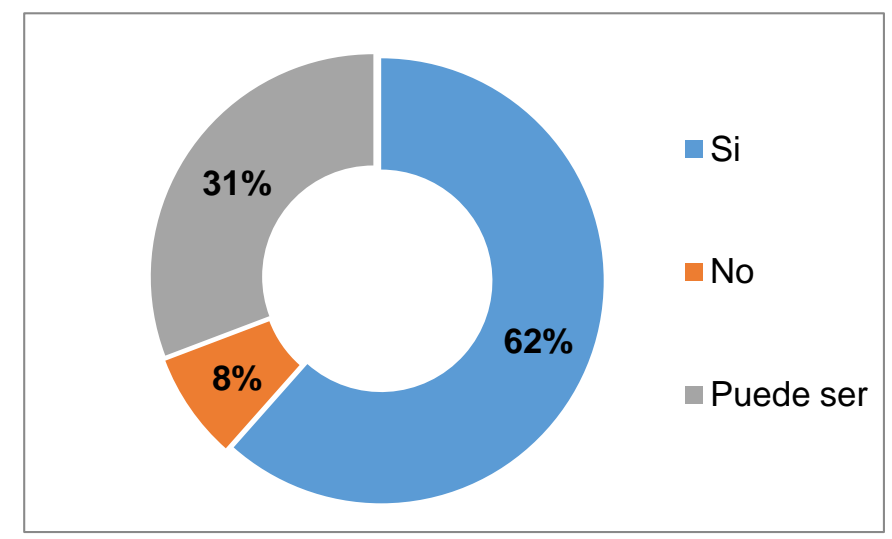

El 62\% de los encuestados coinciden en que lo aprendido en las materias de investigación es suficiente para ejercer en un medio de comunicación; sin embargo, un $31 \%$ indica que puede ser, mientras que el $8 \%$ afirma que con los conocimientos adquiridos aún no se sienten preparados para ejercer en el ámbito laboral.

Es así que los estudios se han transformado constantemente para dar respuesta a las exigencias del mercado laboral y cumplir con verdaderos profesionales; sin embargo, todavía existe un porcentaje que deja mucho que desear ya que el $31 \%$ de los encuestados indican que con lo aprendido en el aula de clases no es suficiente para ejercer en un medio de comunicación.

¿Cuáles son las competencias y habilidades que debe tener un investigador para incorporarse en un medio de comunicación en Ecuador? "En el país no existe mayor apertura a esta rama del periodismo, por temas de presupuestos, tiempos y recursos", así lo indica Allen Panchana, mentalizador del programa Visión 360 de Ecuavisa en una entrevista. El problema principal es que en el país existe un sistema tradicional, donde se priorizan las notas leídas, a diferencia del periodismo de investigación en el que se requiere de un proceso de reflexión al momento de elaborar trabajos periodísticos. Los periodistas deben ser proactivos, pero sobre todo planificadores (Farias, 2018).

Por su parte Reyes en su artículo Percepción del Periodismo Investigativo resalta que se requiere dos componentes "El periodismo de Investigación, como rama activa de la profesión, nació como una necesidad: la de dar mayor perfección y mejor profundización al tratamiento de la información. Por este motivo, una de las tareas principales del periodista responde a fiscalizar y dar a conocer hechos que presentan problemas en el orden democrático actual" (Reyes, 2017). 
Para fortalecer los pensamientos anteriores Levoyer en su artículo sobre Periodismo de investigación, ¿El espacio perdido en el Ecuador? menciona que existen dos motivos principales que hacen que los conceptos de periodismo de investigación en el país se queden en simples utopías. La primera es que el ex-presidente Rafael Correa, desde que inició su mandato en 2007 dividió a la prensa en dos grupos: la pública y la privada. La segunda razón es que los medios tradicionales, en su mayoría, han cerrado sus puertas para informar y narrar los hechos que ocurren en la palestra pública. "No ha sido un proceso rápido, sino paulatino, hasta llegar al 2014 con contados espacios para practicar ese periodismo de datos reveladores que incomodan a quienes tienen cosas que ocultar y que generalmente están en las esferas del poder, cualquiera sea este tipo de poder" (Salas, 2015).

\section{Figura 8}

El periodismo de investigación en Ecuador

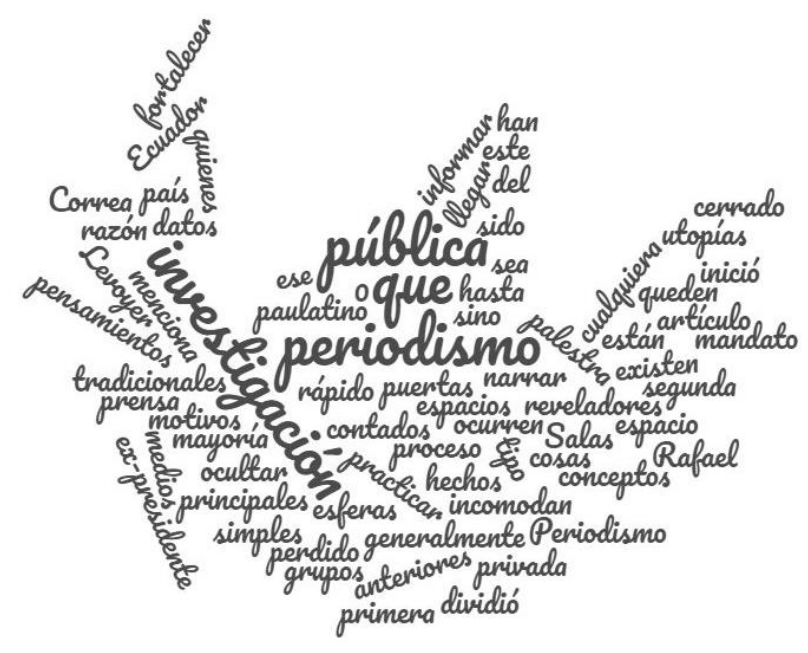

\section{Estrategias en las aulas universitarias}

En análisis sobre los métodos y estrategias a realizar en las aulas universitarias, Juan Varona, realiza una relación de las diferentes técnicas y actividades. Entre las principales el autor se refiere a: realización de productos audiovisuales de investigación, prácticas de producción textual, realización de textos sobre temas reales de la comunidad, talleres, etc. Todo esto depende del docente y de la práctica constante en la asignatura. (Vega y Restrepo, 2015). De esta manera combinar el material teórico con las actividades mencionadas resultaría de gran ayuda para que el estudiante adquiera un aprendizaje significativo donde se combine la teoría y la práctica. "En términos pedagógicos, esto se denomina praxis, momento en el que una teoría se convierte en parte de la experiencia del estudiante" (Díaz, 2009). Es decir, las teorías no son únicamente adquiridas de forma intelectual en las universidades, las ideas son experimentadas en la vida real. Para el presente estudio también se consideraron las opiniones de docentes que imparten las 
materias de periodismo de seis universidades de la ciudad de Guayaquil; de esta manera se evaluaron las nociones adquiridas en el área de periodismo de investigación

\section{Figura 9}

Competencias que debe poseer un profesional

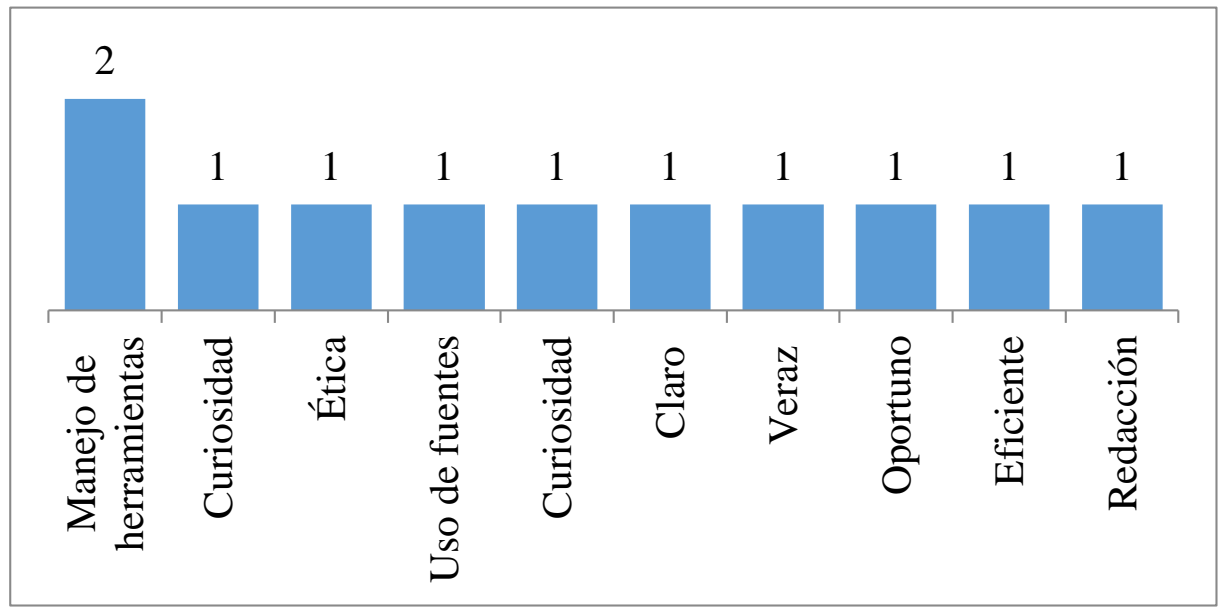

El 100\% de los encuestados opinan que el Periodismo de Investigación es una asignatura que si debe formar parte de la malla académica de todas las universidades. De este rubro $2.19 \%$ mencionaron que dentro de las competencias puntuales que debe poseer un profesional en Periodismo de Investigación predomina el manejo de herramientas de las nuevas tecnologías de comunicación, esto seguido de curiosidad y ética.

\section{Gráfico 10}

La investigación es clave para la enseñanza del periodismo

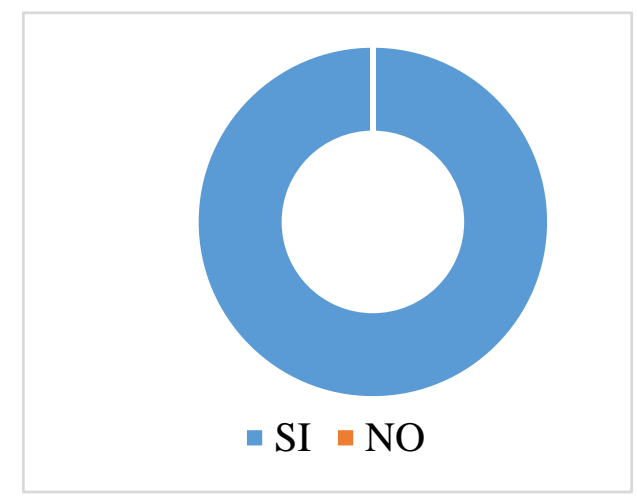


El 100\% de los catedráticos indicaron que realizar una exhaustiva indagación sobre un determinado tema es clave para formar a los futuros profesionales de la comunicación con bases $\mathrm{y}$ herramientas que puedan utilizar a futuro.

Luego de analizar los presentes resultados, no cabe duda de la importancia de la pedagogía al momento de impartir las clases de Periodismo de Investigación. Donde queda claro que el aprendizaje y la enseñanza de esta rama son complejos y no existe una receta establecida para explicarlo, así como indica Vega en su artículo El periodismo: Enseñanza y Aprendizaje. No hay una fórmula en general, ni en particular, hay que analizar la metodología que se utiliza en las clases; donde los estudiantes y docentes deben cumplir un solo objetivo, aprender de ambos para que juntos descubran la verdad, y así "se podría evaluar al docente no por la cantidad de datos almacenados en el cerebro, sino por la facilidad que tenga para guiar a un grupo de estudiantes" (Vega y Restrepo, 2015).

Rodríguez indica que, para producir un verdadero conocimiento se requiere de una forma metodológica que acceda a los datos de forma coherente, lógica y estructurada para que luego el estudiante pueda interpretar y realizar un análisis crítico, reforzando así la capacidad de abordar un tema de relaciones sociales en la práctica. (Rodríguez, Pavón, y Carvajal, 2015).

\section{Estrategias en las aulas de clases}

Para evidenciar dicha práctica a la que se refieren los autores, la Facultad de Comunicaciones de la Universidad de Antioquia publicó la edición número 15 de La Urbe, su periódico de prácticas. Contactar las fuentes y convencerlas para que accedieran a dar entrevistas, les llevó tiempo a la docente encargada y a los estudiantes-reporteros. Los integrantes del equipo periodístico completaron tres días de trabajo de campo, caminando, conversando, interrogando y parapetándose de las balas en los recodos de las calles, casas, tiendas y escuelas de los 19 barrios que conforman la Comuna 13 en Colombia. (Correa, 2013). De esta manera lograron escribir en sus libretas y retener en sus grabadoras y en sus sentidos, la mayoría de los datos, los nombres y las voces que ayudarían a crear los mejores trabajos periodísticos.

Otro caso de éxito sobre la práctica del periodismo de investigación lo realizó la Universidad Complutense de Madrid al implementar un modelo de enlace, en el que un grupo de estudiantes efectuó una amplia investigación dentro de la universidad para poner en opinión pública un asunto verdaderamente escandaloso sobre el aumento desmesurado de dinero a 195 funcionarios, de Administración y Servicios, de dicha universidad, durante varios meses se llevó a cabo la recopilación de información, contrastación de fuentes, entrevistas y encuestas necesarias para finalmente mostrar con evidencias todo lo que ocurría en la institución. (López, 2015). Ambos casos evidencian que se puede realizar proyectos prácticos que involucren la participación de estudiantes y docentes en el periodismo de investigación. 


\section{Conclusiones}

La exhaustiva labor de un periodista de investigación o de un equipo de profesionales puede cambiar el rumbo de un país, de esta manera se mostrará a la sociedad lo que ciertos grupos de poder ansían ocultar, sus ganas por obtener determinados intereses. El verdadero periodismo investigativo, evita mezclar beneficios personales o políticos, busca equidad en una sociedad más justa, con proyectos que develen lo que está pasando dentro de un contexto. Estos procedimientos que se han expuesto en los últimos años ante la palestra pública se han desarrollado gracias al avance que ofrecen las nuevas tecnologías de Información y Comunicación (TIC) y es que a través de ellas se lograron resultados que pudieron transcender fronteras a nivel social y político como los Panamá Papers. "Sus resultados, no sólo por la dimensión cuantitativa sino por las posiciones de los actores involucrados en numerosas naciones, la convierten en la mayor filtración de la historia y de esa manera en un ícono del periodismo global" (Rodríguez, 2016).

Sin embargo, ¿qué se requiere para hacer periodismo de investigación? Ningún proyecto de esta magnitud se realiza de un día para el otro, se necesita tiempo y a su vez dinero que ayude a la gestión del trabajo; pero no siempre se cuenta con los recursos suficientes, así lo expresó en una entrevista Rubén Montoya, profesor de periodismo de Investigación en la Universidad Casa Grande.

Investigación es tiempo y vivimos unos tiempos en los que la celeridad parece ser el sello del trabajo periodístico no está mal hacer las cosas rápido y bien, pero eso está en contra del trabajo de investigación que requiere que es básicamente una segunda mirada, una mucho más profunda (Montoya, 2018).

Todo periodista que realice una labor de investigación exhaustiva debe tener una verdadera vocación por el oficio, paciencia y tenacidad, entre otras habilidades que pueden desarrollarse con la práctica desde la academia, donde es importante inculcarle al futuro comunicador sobre nociones rígidas de ética, responsabilidad periodística y manejo de herramientas adecuadas. "En medio de una eventual avalancha de oferta informativa proveniente de internet, sólo se destacarán aquellos periodistas con training y experiencia” (Fuentes, 2006). Esto sin duda requiere de una visión aguda y capacitación frecuente por parte del profesional de la comunicación, porque quien no esté preparado para asumir los retos que demanda esta carrera, difícilmente tendrá mejores oportunidades en el campo laboral; mientras tanto, grandes temas se seguirán desmantelando para bien de la sociedad gracias a periodistas comprometidos con la búsqueda de la verdad.

Existe un vasto camino por recorrer si de periodismo de investigación se trata sobre todo en el ámbito universitario ya que la formación que reciben los estudiantes es aún deficiente, lo que no implica que sea de mala calidad. Sin embargo, las necesidades del medio en la actualidad demandan ciertas habilidades y destrezas específicas. Lo que concuerda con lo que indicó el periodista argentino Javier Sinay en una entrevista: El periodismo de investigación en 
Latinoamérica aún no ha sido explorado en su totalidad, es un desafío para las nuevas generaciones incorporar las herramientas tecnológicas sin perder la esencia del periodismo y la investigación.

Los estudiantes deben estar preparados para enfrentar la realidad del periodismo de investigación, es un reto que va de la mano con la preparación académica en las aulas de clases como lo indica Rodríguez: "Solamente con proyectos de investigación bien concebidos desde la academia, los programas de formación en periodismo y la práctica constante de los futuros profesionales podrán corresponder a los conocimientos, habilidades y actitudes que reclama la sociedad" (Rodríguez, Pavón, y Carvajal, 2015).

Para cumplir con este objetivo se necesita hacer prácticas profesionales y tener una exigente formación académica, con el fin de desarrollar habilidades investigativas que les permitan desempeñarse acorde a las necesidades del medio. Para ello es necesario que los docentes incorporen nuevas estrategias como: la realización de trabajos que involucren investigación de campo y proyectos que les permitan obtener trabajos periodísticos de buena calidad. El panorama en los planes de estudios en las universidades aún no satisface las necesidades de los estudiantes, como se evidencia en las encuestas, aún existe un alto porcentaje de estudiantes que no se sienten completamente preparados para laborar en un medio de comunicación. Es recomendable que las mallas curriculares aborden más materias que refuercen el Periodismo de Investigación direccionado en diversos formatos y plataformas, para estar preparados a los constantes cambios que desde en el ámbito comunicacional impone la realidad del periodismo.

\section{Referencias Bibliográficas}

Álvarez, M. A. (2015). El papel de la investigación en la prensa escrita cuencana. Cuenca: Universidad de Cuenca.

Campbell.

(1994).

Obtenido

de http://www.razonypalabra.org.mx/N/N81/V81/11_McPhail_V81.pdf

Correa, C. (2013). El laboratorio de periodismo. La práctica universitaria de la idea fundacional de Pulitzer. Obtenido de https://www.redalyc.org/articulo.oa?id=77428608003

Díaz, C. (2009). Los procesos de enseñanza-aprendizaje en el aula universitaria: una mirada desde las creencias de académicos de trabajo social y periodismo. Obtenido de https://scielo.conicyt.cl/scielo.php?script=sci_arttext\&pid=S071807052009000100011\&lng=es\&nrm=i\&tlng=es

Fanger, E. M. (2012). Periodismo de investigación: El caso Wal Mart de México. Razón y Palabra, 9.

Farias, T. (2018 de noviembre de 2018). Entrevista sobre periodismo de investigación- Allen Panchana. (T. Farias, \& D. Gutiérrez, Entrevistadores)

Fuentes, C. (2006). Obtenido de http://www.cuadernos.info/index.php/CDI/article/view/126/118

Esta obra se comparte bajo la licencia Creative Common Atribución-No Comercial 4.0 International (CC BY-NC 4.0) Revista de la Universidad Internacional del Ecuador. URL: https://www.uide.edu.ec/ 
González, M. (11 de enero de 2019). El Comercio. Obtenido de https://www.elcomercio.com/actualidad/ecuador-deterioro-condiciones-julianassange.html

Guzmán, V. (2016). El periodismo como poder protagonista de cambios sociales. Estudio de casos específicos: Watergate y Wikileaks.

Humanes, M., \& Roses, S. (2014). Valoración de los estudiantes sobre la enseñanza del Periodismo en España. Obtenido de https://www.redalyc.org/pdf/158/15830197020.pdf

Hunter, M. L. (2013). La Investigación a partir de historias: manual para periodistas de investigación. Montevideo: UNESCO.

Leñero, V., \& Marín, C. (1986). Redalyc. Obtenido de Redalyc: https://concienciaperiodistica.files.wordpress.com/2015/05/lec2b1ero-vicente-y-carlosmarin-manual-de-periodismo.pdf

Lina Leal, S. T. (2017). Los avatares del periodismo de investigación en Colombia. Argumentos.

Llanca, M., \& Reyes, N. (2017). Percepción del Periodismo Investigativo de los estudiantes de la Región de Valparaíso. Obtenido de http://opac.pucv.cl/pucv_txt/txt2000/UCC2282_01.pdf

López, J. (30 de octubre de 2015). El innovador modelo del enlace y el periodismo de investigación. $\quad$ Obtenido file://C:/Users/Invitado/Downloads/PAPER\%204\%20MODELO\%20.pdf

Montoya, R. (19 de noviembre de 2018). (D. Gutiérrez, Entrevistador)

Muraro. (1997). Obtenido de file:///C:/Users/Nicole/Downloads/3629-13595-1-PB.pdf

Muraro. (2000). Obtenido de http://www.razonypalabra.org.mx/anteriores/n43/vlongo.html

Orellana, G. (2018). Proyecto de Inserción Social y Educativa en Barrios de PAz. Guayaquil.

Poveda, S. (19 de noviembre de 2018). (D. Gutiérrez, Entrevistador)

Requejo, J. L. (2011). El legado de los Muckrakers. España: Universidad Carlos III de Madrid.

Reyes, G. (2005). Centro de Periodistas de Investigación. Recuperado el 2018, de Centro de Periodistas de Investigación: http://investigacion.org.mx/libro5.html

Reyes, L. \&. (2017). Percepción del Periodismo Investigativo de los estudiantes de la Región de Valparaíso, Obtenido de http://opac.pucv.cl/pucv_txt/txt-2000/UCC2282_01.pdf

Rodríguez, B., Pavón, L. B., \& Carvajal, R. S. (diciembre de 2015). Enseñanza, investigación y praxis profesional: Desafios de la formación del periodista. Obtenido de Razón y Palabra: https://www.redalyc.org/pdf/1995/199543036009.pdf

Rodríguez, M. E. (noviembre de 2016). Redcom. Obtenido de Redcom: file:///C:/Users/Nicole/Documents/periodismo\%20de\%20investigación/PAPERS\%20VIA LIDAD\%20SOBRE\%20EL\%20PERIODISMO\%20DE\%20INVESTIGACIÓN\%20PERI ODISTAS\%20EN\%20LA\%20ERA\%20DIGITAL/Papeles\%20del\%20lavado\%20y\%20p eriodismo\%20de\%20investigación.pdf

Salas, S. L. (2015). Periodismo de investigación ¿El espacio perdido en el Ecuador? Obtenido de Boletin informativo Spodylus: http://www.uasb.edu.ec/documents/10181/423786/SaudiaLevoyer+\%5BUnidadesInvesti gacion\%5D.pdf/19f2f010-318a-4553-8061-2b3e645273e7 
Samaniego, G. (12 de diciembre de 2018). Entrevista a Javier Sinay, periodista argentino. (G. Samaniego, Entrevistador)

Santoro, D. (2004). Técnicas de Investigación. México.

Soto, C. M. (2013). El laboratorio de periodismo. La práctica universitaria de la idea fundacional de Pulitzer. Obtenido de https://www.redalyc.org/articulo.oa?id=77428608003

Starkman, D. (2014). El perro guardián que no ladró. La crisis financiera y la desaparición del periodismo de investigación. Nueva York: Columbia University Press.

Universidad Internacional del Ecuador. (25 de noviembre de 2018). Encuestas sobre periodismo de investigación en las universidades. Guayaquil.

Valarezo, L. (noviembre de 2018). Periodismo de Investigación. (K. Rojas, Entrevistador)

Vega, M. F., \& Restrepo, M. J. (2015). PERIODISMO: Enseñanza y aprendizaje. Luciérnaga, 81. Obtenido de http://www.politecnicojic.edu.co/images/downloads/publicaciones/revistaluciernaga/luciernaga-14/pdf/periodismo.pdf

Walker, M. (05 de noviembre de 2015). Centro de Periodismo Investigativo. Obtenido de http://periodismoinvestigativo.com/2015/11/marina-walker-guevara-y-las-historiasimparables/

Wharton, U. d. (06 de junio de 2012). América economía. Obtenido de https://mba.americaeconomia.com/articulos/reportajes/las-implicaciones-del-caso-desoborno-de-walmart

Woodward, B. (2016). Google Academics. Obtenido de file:///C:/Users/Nicole/Documents/periodismo\%20de\%20investigación/PAPERS\%20VIA LIDAD\%20SOBRE\%20EL\%20PERIODISMO\%20DE\%20INVESTIGACIÓN\%20PERI ODISTAS\%20EN\%20LA\%20ERA\%20DIGITAL/tesis\%201.pdf 